\title{
BEHAVIOR OF HOLOMORPHIC MAPPINGS ON $p$-COMPACT SETS IN A BANACH SPACE
}

\author{
RICHARD M. ARON, ERHAN ÇALIŞKAN, DOMINGO GARCÍA, \\ AND MANUEL MAESTRE
}

\begin{abstract}
We study the behavior of holomorphic mappings on $p$-compact sets in Banach spaces. We show that the image of a $p$-compact set by an entire mapping is a $p$-compact set. Some results related to the localization of $p$-compact sets in the predual of homogeneous polynomials are also obtained. Finally, the "size" of $p$-compactness of the image of the unit ball by $p$-compact linear operators is studied.
\end{abstract}

\section{INTRODUCTION}

Motivated by the well-known Grothendieck characterization of compact sets [10, Sinha and Karn [16] introduced the notion of $p$-compactness. Their work provided motivation for many papers related to the $p$-compactness notion in a number of different directions. We mention [1, [2], 44, [5], 7], 8], 11], 16], and [17.

In this paper we study the behavior of holomorphic mappings on $p$-compact sets. It is a simple fact that the image of a compact set by a continuous function is a compact set, but we shall observe that the analogous result is not always true if one considers $p$-compact sets. With this in mind, it is natural to ask what happens if we consider holomorphic mappings and, in particular, polynomials. We show that the image of a $p$-compact set by an entire mapping is always $p$-compact.

The paper is organized as follows: In section 2 we establish our notation and definitions. In section 3 we study the behavior of holomorphic mappings on $p$ compact sets. First we show that the image of a $p$-compact set by a polynomial is always a $p$-compact set. In fact, this result is already known, with a proof by the first author and P. Rueda [2] that uses tensor product methods. However, the machinery that we develop here allows us to obtain a positive answer for entire mappings. In section 4 we get a result related to the localization of $p$-compact sets in symmetric tensor products of a Banach space with itself, which runs parallel to a result that Ryan [14] obtained for compact sets. Furthermore, we answer in the negative the following question: Is the image of a $p$-compact set, by an $m$-homogeneous polynomial, $p / m$-compact? To do this we give an example of a homogeneous polynomial $P$ and a $p$-compact set $K$ whose image $P(K)$ is not a

Received by the editors July 24, 2013 and, in revised form, April 15, 2014 and May 26, 2014.

2010 Mathematics Subject Classification. Primary 46G20; Secondary 46B28, 46G25.

Key words and phrases. Banach spaces, $p$-compact sets, homogeneous polynomials, holomorphic mappings.

The first, third and fourth authors were supported by MICINN Project MTM2011-22417 and by MINECO MTM2014-57838-C2-2-P. The third and fourth authors were also supported by Prometeo II/2013/013. The second author was supported by TÜBİTAK - The Scientific and Technological Research Council of Turkey. 
$q$-compact set for any $1 \leq q<p$. As a converse, we show that given a $p$-compact subset of the image of an $m$-homogeneous polynomial, in general it is not possible to find a $p$-compact set whose image contains the given $p$-compact set. In section 5 we show that the "size" (in the sense that we will make precise there) of $p$-compactness of the image of the unit ball by $p$-compact linear operators between $l_{p}$ spaces can be arbitrarily large.

\section{Notation AND DEFinitions}

The symbol $\mathbb{C}$ represents the field of all complex numbers, $\mathbb{N}$ represents the set of all positive integers, and $\mathbb{N}_{0}=\mathbb{N} \cup\{0\}$.

The letters $X$ and $Y$ will always represent complex Banach spaces. The letter $U$ denotes a nonvoid open subset of $X$ and the symbol $B_{X}$ represents the open unit ball of $X$. The symbol $\bar{B}_{X}$ represents the closed unit ball of $X$.

Given a subset $M$ of $X$, the symbol $\left.f\right|_{M}$ will denote the restriction of a mapping $f: U \longrightarrow Y$ to the subset $M \subset U$, the symbol $I_{M}$ will denote the identity mapping on $M$, and for any topology $\tau$ on $X, \bar{M}^{\tau}$ will denote the $\tau$-closure of $M$ in $X$.

For each integer $n \in \mathbb{N}$, let $\mathcal{L}\left(X_{1}, \ldots, X_{n} ; Y\right)$ be the Banach space of all continuous $n$-linear mappings $A: X_{1} \times \ldots \times X_{n} \longmapsto Y$, endowed with the sup norm $\|A\|=$ $\sup \left\{\left\|A\left(x_{1}, \ldots, x_{n}\right)\right\|:\left\|x_{i}\right\| \leq 1, i=1, \ldots, n\right\}$. When $X_{1}=\cdots=X_{n}=X$ we write $\mathcal{L}\left({ }^{n} X ; Y\right)$ instead of $\mathcal{L}(\underbrace{X, \ldots, X}_{n \text { times }} ; Y)$, and in particular, $\mathcal{L}\left({ }^{1} X ; Y\right)=\mathcal{L}(X ; Y)$.

The subspace of all compact operators of $\mathcal{L}(X ; Y)$ will be denoted by $\mathcal{K}(X ; Y)$.

Given a continuous $n$-linear mapping $A \in \mathcal{L}\left({ }^{n} X ; Y\right)$, the map $P: X \longrightarrow Y$, defined by $P(x)=A(\underbrace{x, \ldots, x}_{n \text { times }})$ for every $x \in X$, is said to be a continuous $n$ homogeneous polynomial. $\mathcal{P}\left({ }^{n} X ; Y\right)$ will denote the vector space of all continuous $n$-homogeneous polynomials from $X$ into $Y$, which is a Banach space with norm $\|P\|=\sup \{\|P(x)\|:\|x\| \leq 1\}$. The constant functions from $X$ into $Y$ will be denoted by $\mathcal{P}\left({ }^{0} X ; Y\right)$. When $Y=\mathbb{C}$ we will write $\mathcal{P}\left({ }^{n} X\right)$ instead of $\mathcal{P}\left({ }^{n} X ; \mathbb{C}\right)$.

If $A$ is a multilinear mapping and $P$ is the polynomial generated by $A$, we write $P=\widehat{A}$. Conversely, given a polynomial $P \in \mathcal{P}\left({ }^{n} X ; Y\right)$, there is a unique symmetric continuous $n$-linear mapping $\stackrel{\vee}{P} \in \mathcal{L}\left({ }^{n} X ; Y\right)$ such that $P(x)=\stackrel{\vee}{P}(\underbrace{x, \ldots, x}_{n \text { times }})$. It is well known that the correspondence $A \longleftrightarrow \widehat{A}$ is a topological isomorphism between $\mathcal{L}^{s}\left({ }^{n} X ; Y\right)$, the space of all symmetric continuous $n$-linear mappings from $X$ to $Y$, and $\mathcal{P}\left({ }^{n} X ; Y\right)$ (see, for example, [13, Theorem 2.2]).

A mapping $P: X \longrightarrow Y$ is said to be a continuous polynomial if it can be represented as a sum $P=P^{0}+P^{1}+\cdots+P^{n}$, with $P^{j} \in \mathcal{P}\left({ }^{j} X ; Y\right)$ for $j=0,1, \ldots, n$. By $\mathcal{P}(X ; Y)$ we will denote the vector space of all continuous polynomials from $X$ into $Y$.

Let $X$ be a Banach space, let $1 \leq p \leq \infty$, with the conjugate index $p^{\prime}$ given by $\frac{1}{p}+\frac{1}{p^{\prime}}=1$ (where $p^{\prime}=1$ if $\left.p=\infty\right)$, let $\ell_{p}(X)(1 \leq p<\infty)\left(\operatorname{resp}\right.$., $\left.\ell_{\infty}(X)\right)$ denote the set of all sequences $\left(x_{n}\right)_{n=1}^{\infty}$ in $X$ such that $\sum_{n=1}^{\infty}\left\|x_{n}\right\|^{p}<\infty$ (resp., $\left(x_{n}\right)_{n}$ is bounded), and let $c_{0}(X)$ denote the set of all sequences $\left(x_{n}\right)_{n=1}^{\infty}$ in $X$ such that $x_{n} \longrightarrow 0$ in $X$. Then a set $K \subset X$ is said to be relatively $p$-compact if there is a sequence $\left(x_{n}\right)_{n=1}^{\infty}$ in $\ell_{p}(X)\left(\left(x_{n}\right)_{n=1}^{\infty}\right.$ in $c_{0}(X) \subset \ell_{\infty}(X)$ if $\left.p=\infty\right)$ such that $K \subset\left\{\sum_{n=1}^{\infty} a_{n} x_{n}:\left(a_{n}\right)_{n=1}^{\infty} \in \bar{B}_{\ell_{p^{\prime}}}\right\}$. A relatively $p$-compact and closed set 
will be called $p$-compact. We denote this last set by $p$-co $\left\{\left(x_{n}\right)_{n=1}^{\infty}\right\}$ and we will call it a fundamental $p$-compact set since these sets form a fundamental system of $p$-compact sets of $X$.

From Grothendieck's characterization of compact sets (see [10], 12, Proposition 1.e.2]), a subset $K$ of a Banach space $X$ is relatively compact if and only if there is a sequence $\left(x_{n}\right)_{n=1}^{\infty}$ in $c_{0}(X)$ such that $K \subset\left\{\sum_{n=1}^{\infty} a_{n} x_{n}: \sum_{n=1}^{\infty}\left|a_{n}\right| \leq 1\right\}$. Thus, by the above definition one can consider compact sets as $\infty$-compact. Also, note that $p$-compact sets are $q$-compact if $1 \leq p \leq q \leq \infty$.

We refer to [9] or [13] for the properties of polynomials and holomorphic mappings in infinite dimensional spaces, and to [12] for the theory of Banach spaces.

\section{The Stability of $p$-COMPACT SETS}

First of all we observe that the image of a $p$-compact set by a continuous function need not be a $p$-compact set. In fact, we have the following example.

Example 3.1. Let $1<p<\infty$ and let $\left(a_{n}\right)_{n=1}^{\infty} \in c_{0} \backslash \ell_{p}$. Let $f: \mathbb{R} \longrightarrow \ell_{p}$ be defined by

$f(t)= \begin{cases}0 & \text { if } t \leq 0, \\ (n+1)(1-n t) a_{n+1} e_{n+1}+n((n+1) t-1) a_{n} e_{n} & \text { if } t \in\left[\frac{1}{n+1}, \frac{1}{n}\right] \text { for } n \in \mathbb{N}, \\ a_{1} e_{1} & \text { if } t \geq 1,\end{cases}$

where $\left(e_{n}\right)_{n=1}^{\infty}$ is the canonical basis of $\ell_{p}$. Observe that $f$ is continuous and that $f([0,1]) \supset\left(a_{n} e_{n}\right)_{n=1}^{\infty}$. By [1, Example 1] we know that the set $\left(a_{n} e_{n}\right)_{n=1}^{\infty}$ is a relatively compact set in $\ell_{p}$ which is not $p$-compact. Consequently $f([0,1])$ cannot be a $p$-compact set in $\ell_{p}$. But, since the interval $[0,1]$ is a $p$-compact subset of $\mathbb{R}$ (see e.g. [1, Proposition 2]), we are done.

On the other hand, contrary to the non-linear case, it is easy to show that the image of a $p$-compact set by a continuous linear operator is a $p$-compact set.

In [2], using linearization of homogeneous polynomials and tensor products, the first author and P. Rueda show that the image of a $p$-compact set by a homogeneous polynomial is also $p$-compact. For a more general result see [3, Theorem 3.5]. Below we obtain the same result in a different way. Although our proof is longer than that in [2], the proof that we will present has the advantage of yielding, as a bonus, a proof showing that the same is true for entire mappings.

Lemma 3.2. Let $X$ and $Y$ be Banach spaces, and let $n \in \mathbb{N}, P \in \mathcal{P}\left({ }^{n} X ; Y\right)$ and $1 \leq p \leq \infty$. If $\left(x_{k}\right)_{k=1}^{\infty} \in \ell_{p}(X)$, then the set $P\left(\left\{\sum_{k=1}^{\infty} \alpha_{k} x_{k}:\left(\alpha_{k}\right)_{k=1}^{\infty} \in \bar{B}_{\ell_{p^{\prime}}}\right\}\right)$ is a p-compact subset of $Y$.

Proof. Since $\infty$-compact sets are compact, we shall give a proof for the case $p>1$. Minor modifications yield a proof for the $p=1$ case.

Let $n \in \mathbb{N}$ and $P \in \mathcal{P}\left({ }^{n} X ; Y\right)$. Let $\left(x_{k}\right)_{k=1}^{\infty}$ be a sequence in $\ell_{p}(X)$. To show that the image $P\left(\left\{\sum_{k=1}^{\infty} \alpha_{k} x_{k}:\left(\alpha_{k}\right)_{k=1}^{\infty} \in \bar{B}_{\ell_{p^{\prime}}}\right\}\right)$ is $p$-compact, let us take an element $x \in\left\{\sum_{k=1}^{\infty} \alpha_{k} x_{k}:\left(\alpha_{k}\right)_{k=1}^{\infty} \in \bar{B}_{\ell_{p^{\prime}}}\right\}$ with $x=\sum_{k=1}^{\infty} \alpha_{k} x_{k}$ for some $\left(\alpha_{k}\right)_{k=1}^{\infty} \in \bar{B}_{\ell_{p^{\prime}}}$. 
Let $h \in \mathbb{N}$ be any integer. By the Leibniz formula we have that

$$
\begin{aligned}
& P\left(\sum_{k=1}^{h} \alpha_{k} x_{k}\right)=\sum_{\substack{l_{1}+\cdots+l_{h}=n \\
l_{j} \in\{0,1, \ldots, n\}}} \frac{n !}{l_{1} ! \cdots l_{h} !} \alpha_{1}^{l_{1}} \cdots \alpha_{h}^{l_{h}} \stackrel{\vee}{P}\left(x_{1}^{l_{1}}, \ldots, x_{h}^{l_{h}}\right) \\
& \quad=\sum_{\substack{l_{1}+\cdots+l_{h}=n \\
l_{j} \in\{0,1, \ldots, n\}}}\left(\frac{n !}{l_{1} ! \cdots l_{h} !}\right)^{1 / p^{\prime}} \alpha_{1}^{l_{1}} \cdots \alpha_{h}^{l_{h}}\left(\frac{n !}{l_{1} ! \cdots l_{h} !}\right)^{1 / p} \stackrel{\vee}{P}\left(x_{1}^{l_{1}}, \ldots, x_{h}^{l_{h}}\right),
\end{aligned}
$$

where $\stackrel{\vee}{P}$ is the continuous symmetric $n$-linear mapping corresponding to the $n$ homogeneous polynomial $P$. Note that, by the multinomial theorem,

$$
\begin{aligned}
& \left(\sum_{\substack{l_{1}+\cdots+l_{h}=n \\
l_{j} \in\{0,1, \ldots, n\}}}\left[\left(\frac{n !}{l_{1} ! \cdots l_{h} !}\right)^{1 / p^{\prime}}\left|\alpha_{1}^{l_{1}}\right| \cdots\left|\alpha_{h}^{l_{h}}\right|\right]^{p^{\prime}}\right)^{1 / p^{\prime}} \\
& =\left(\sum_{\substack{l_{1}+\cdots+l_{h}=n \\
l_{j} \in\{0,1, \ldots, n\}}} \frac{n !}{l_{1} ! \cdots l_{h} !}\left|\alpha_{1}^{p^{\prime}}\right|^{l_{1}} \cdots\left|\alpha_{h}^{p^{\prime}}\right|^{l_{h}}\right)^{1 / p^{\prime}} \\
& =\left(\sum_{k=1}^{h}\left|\alpha_{k}\right|^{p^{\prime}}\right)^{n / p^{\prime}} \leq\left\|\left(\alpha_{k}\right)_{k \in \mathbb{N}}\right\|_{p^{\prime}}^{n} \leq 1
\end{aligned}
$$

and analogously

$$
\begin{aligned}
& \left(\sum_{\substack{l_{1}+\cdots+l_{h}=n \\
l_{j} \in\{0,1, \ldots, n\}}}\left[\left(\frac{n !}{l_{1} ! \cdots l_{h} !}\right)^{1 / p}\left\|\stackrel{\vee}{P}\left(x_{1}^{l_{1}}, \ldots, x_{h}^{l_{h}}\right)\right\|^{p}\right]^{1 / p}\right. \\
\leq & \left(\sum_{\substack{l_{1}+\cdots+l_{h}=n \\
l_{j} \in\{0,1, \ldots, n\}}} \frac{n !}{l_{1} ! \cdots l_{h} !}\|\stackrel{\vee}{P}\| \|^{p}\left(\left\|x_{1}\right\|^{p}\right)^{l_{1}} \ldots\left(\left\|x_{h}\right\|^{p}\right)^{l_{h}}\right)^{1 / p} \\
= & \|\stackrel{\vee}{P}\|\left(\sum_{k=1}^{h}\left\|x_{k}\right\|^{p}\right)^{n / p} \leq\|\stackrel{\vee}{P}\|\left(\sum_{k=1}^{\infty}\left\|x_{k}\right\|^{p}\right)^{n / p}<\infty .
\end{aligned}
$$

Now we claim that if $\left(\alpha_{k}\right)_{k=1}^{\infty} \in \bar{B}_{\ell_{p^{\prime}}}$ we have

$$
P\left(\sum_{k=1}^{\infty} \alpha_{k} x_{k}\right)=\sum_{\substack{\sum_{j=1}^{\infty} l_{j}=n \\ l_{j} \in\{0,1, \ldots, n\}}} \frac{n !}{l_{1} ! \cdots l_{j} ! \ldots} \prod_{j=1}^{\infty} \alpha_{j}^{l_{j}} \stackrel{\vee}{P}\left(x_{1}^{l_{1}}, \ldots, x_{j}^{l_{j}}, \ldots\right) .
$$

Let

$$
\mathbb{A}_{n}:=\left\{\left(l_{j}\right)_{j=1}^{\infty} \in\{0, \ldots, n\}^{\mathbb{N}}: \sum_{j=1}^{\infty} l_{j}=n\right\}
$$


and let $J$ be a finite subset of $\mathbb{A}_{n}$. Then there exists an integer $h \in \mathbb{N}$ such that

$$
J \subset \mathbb{B}_{n}^{h}:=\left\{\left(l_{j}\right)_{j=1}^{h} \in\{0, \ldots, n\}^{h}: \sum_{j=1}^{h} l_{j}=n\right\} \times\{0\}^{\mathbb{N}} .
$$

Thus

$$
\begin{aligned}
\sum_{l \in J} \frac{n !}{l_{1} ! \cdots l_{j} ! \cdots} \prod_{j=1}^{\infty}\left|\alpha_{j}\right|^{l_{j}}\left\|\stackrel{\vee}{P}\left(x_{1}^{l_{1}}, \ldots, x_{j}^{l_{j}}, \ldots\right)\right\| \\
\leq \sum_{\substack{l_{1}+\cdots+l_{h}=n \\
l_{j} \in\{0,1, \ldots, n\}}} \frac{n !}{l_{1} ! \cdots l_{h} !}\left|\alpha_{1}\right|^{l_{1}} \cdots\left|\alpha_{h}\right|^{l_{h}}\left\|\stackrel { \vee } { P } \left|\left\||| x_{1}\right\|^{l_{1}} \ldots\left\|x_{h}\right\|^{l_{h}}\right.\right. \\
\quad=\|\stackrel{\vee}{P}\|\left(\sum_{k=1}^{h}\left|\alpha_{k}\right|\left\|x_{k}\right\|\right)^{n} \leq\|\stackrel{\vee}{P}\|\left(\sum_{k=1}^{\infty}\left|\alpha_{k}\right|^{p^{\prime}}\right)^{n / p^{\prime}}\left(\sum_{k=1}^{\infty}\left\|x_{k}\right\|^{p}\right)^{n / p} \\
\leq\|\stackrel{\vee}{P}\|\left\|\left(x_{k}\right)_{k=1}^{\infty}\right\|_{p}^{n}<\infty,
\end{aligned}
$$

which shows that the series on the right-hand side of (3) is absolutely summable. In particular, the sequence $\left(\sum_{l \in \mathbb{B}_{n}^{h}}\left(\begin{array}{c}n \\ l\end{array}\right) \alpha^{l} \stackrel{\vee}{P}\left(x^{l}\right)\right)_{h \in \mathbb{N}}$ converges to $\sum_{l \in \mathbb{A}_{n}}\left(\begin{array}{c}n \\ l\end{array}\right) \alpha^{l} \stackrel{\vee}{P}\left(x^{l}\right)$, where we use the notation: $\alpha=\left(\alpha_{k}\right)_{k=1}^{\infty}, x=\left(x_{k}\right)_{k=1}^{\infty}$, and

$$
\left(\begin{array}{c}
n \\
l
\end{array}\right)=\frac{n !}{\prod_{j=1}^{\infty} l_{j} !}, \quad \alpha^{l}=\prod_{j=1}^{\infty} \alpha_{j}^{l_{j}}, \quad \stackrel{\vee}{P}_{n}\left(x^{l}\right)=\stackrel{\vee}{P_{n}}\left(x_{1}^{l_{1}}, \ldots, x_{j}^{l_{j}}, \ldots\right) .
$$

Since the sequence $\left(P\left(\sum_{k=1}^{h} \alpha_{k} x_{k}\right)\right)_{h \in \mathbb{N}}$ converges to $P\left(\sum_{k=1}^{\infty} \alpha_{k} x_{k}\right)$ and

$$
P\left(\sum_{k=1}^{h} \alpha_{k} x_{k}\right)=\sum_{l \in \mathbb{B}_{n}^{h}}\left(\begin{array}{l}
n \\
l
\end{array}\right) \alpha^{l} \stackrel{\vee}{P}\left(x^{l}\right),
$$

it follows that

$$
P\left(\sum_{k=1}^{\infty} \alpha_{k} x_{k}\right)=\sum_{l \in \mathbb{A}_{n}}\left(\begin{array}{l}
n \\
l
\end{array}\right) \alpha^{l} \stackrel{\vee}{P}\left(x^{l}\right)=\sum_{l \in \mathbb{A}_{n}}\left(\begin{array}{l}
n \\
l
\end{array}\right)^{1 / p^{\prime}} \alpha^{l}\left(\begin{array}{l}
n \\
l
\end{array}\right)^{1 / p} \stackrel{\vee}{P}\left(x^{l}\right) .
$$

Noting that $\mathbb{A}_{n}$ is a countable set and by using (11) and (2), respectively, we have

$$
\left(\left(\begin{array}{l}
n \\
l
\end{array}\right)^{1 / p^{\prime}} \alpha^{l}\right)_{l \in \mathbb{A}_{n}} \in \bar{B}_{\ell_{p^{\prime}}} \text { and }\left(\left(\begin{array}{c}
n \\
l
\end{array}\right)^{1 / p} \stackrel{\vee}{P}\left(x^{l}\right)\right)_{l \in \mathbb{A}_{n}} \in \ell_{p}(Y),
$$

which completes the proof.

Corollary 3.3. Let $X$ and $Y$ be Banach spaces and let $P \in \mathcal{P}(X ; Y)$. If $K \subset X$ is a p-compact set, then the set $P(K) \subset Y$ is a p-compact set.

Proof. Let $K$ be a $p$-compact subset of $X$. Then, there is a sequence $\left(x_{k}\right)_{k=1}^{\infty} \in$ $\ell_{p}(X)$ such that $K \subset\left\{\sum_{k=1}^{\infty} \alpha_{k} x_{k}:\left(\alpha_{k}\right)_{k=1}^{\infty} \in \bar{B}_{\ell_{p^{\prime}}}\right\}$. By the previous lemma the set $P(K)$ is a $p$-compact subset of $Y$, for every homogeneous polynomial $P \in$ $\mathcal{P}\left({ }^{n} X ; Y\right)$ and for every $n \in \mathbb{N}$. Since a finite sum of $p$-compact sets is $p$-compact, we are done. 
Now we are going to use our "quantitative" version of the proof that the image of a $p$-compact set by a polynomial is also $p$-compact in order to prove the analogue of Corollary 3.3 for entire functions.

Let $X$ be a Banach space and let $U$ be an open subset of $X$. We denote by $\mathcal{H}(U ; Y)$ the space of all holomorphic functions $f$ from $U$ into $Y$. When $Y=\mathbb{C}$ we write $\mathcal{H}(U)$ instead of $\mathcal{H}(U ; \mathbb{C})$.

We will need the following.

Lemma 3.4. Let $X$ and $Y$ be Banach spaces, and let $U$ be a balanced open subset of $X$ and $s>0$ satisfying $s B_{X} \subset U$. Consider a sequence $\left(x_{k}\right)_{k=1}^{\infty}$ in $\ell_{p}(X)$ with p-norm $\left\|\left(x_{k}\right)_{k=1}^{\infty}\right\|_{p}<\frac{s}{e}$. Finally, let $\epsilon>0$ and $R>e$ such that

$$
(1+\epsilon)^{1 / p}\left(\frac{R}{e}\right)^{1 / p^{\prime}}\left\|\left(x_{k}\right)_{k=1}^{\infty}\right\|_{p}<\frac{s}{e} .
$$

We denote $M=2\left(\frac{e}{R-e}\right)^{1 / p^{\prime}} \frac{1}{\epsilon^{1 / p}}+2$. Then, for every $K \subset p$-co $\left\{\left(x_{k}\right)_{k=1}^{\infty}\right\}$ and every $f \in \mathcal{H}(U ; Y)$ bounded on $s B_{X}$ it holds that $f(K)$ is relatively $p$-compact. Moreover, there exists a sequence $\left(y_{j}\right)$ in $\ell_{p}(Y)$ (that depends on $f$ ) such that

$$
f(K) \subset p-c o\left\{\left(y_{j}\right)_{j=1}^{\infty}\right\}
$$

and

$$
\left\|\left(y_{j}\right)_{j=1}^{\infty}\right\|_{p} \leq M\|f\|_{s B_{X}} .
$$

Observe that $M$ depends neither on $f$ nor on $\left(y_{j}\right)$.

Proof. As in the polynomial case, we give a proof for the case $p>1$ since the proof for the case $p=1$ is similar.

We will show that the set

$$
f\left(\left\{\sum_{k=1}^{\infty} \alpha_{k} x_{k}:\left(\alpha_{k}\right)_{k=1}^{\infty} \in \bar{B}_{\ell_{p^{\prime}}}\right\}\right)
$$

is relatively $p$-compact.

Let $f=\sum_{n=0}^{\infty} P_{n}$ be the Taylor series expansion of $f$ about 0 , with $P_{n} \in$ $\mathcal{P}\left({ }^{n} X ; Y\right)$ for every $n \in \mathbb{N}_{0}$.

Let $x \in\left\{\sum_{k=1}^{\infty} \alpha_{k} x_{k}:\left(\alpha_{k}\right)_{k=1}^{\infty} \in \bar{B}_{\ell_{p^{\prime}}}\right\}$. By (3) we have that

$$
f(x)-f(0)=\sum_{n=1}^{\infty} P_{n}(x)=\sum_{n=1}^{\infty} \sum_{l \in \mathbb{A}_{n}}\left(\begin{array}{l}
n \\
l
\end{array}\right) \alpha^{l} \stackrel{\vee}{P_{n}}\left(x^{l}\right),
$$

where we use the same notation as in Lemma 3.2 .

Since we have chosen $\varepsilon>0$ and $R>e$ such that

$$
(1+\epsilon)^{1 / p}\left(\frac{R}{e}\right)^{1 / p^{\prime}} e\left(\sum_{k=1}^{\infty}\left\|x_{k}\right\|^{p}\right)^{1 / p}<s
$$

we can write

$$
f(x)-f(0)=\sum_{n=1}^{\infty} \sum_{l \in \mathbb{A}_{n}}\left(\left(\frac{e}{R}\right)^{n / p^{\prime}}\left(\begin{array}{l}
n \\
l
\end{array}\right)^{1 / p^{\prime}} \alpha^{l}\right)\left(\left(\begin{array}{l}
n \\
l
\end{array}\right)^{1 / p}\left(\frac{R}{e}\right)^{n / p^{\prime}} \stackrel{\vee}{P_{n}}\left(x^{l}\right)\right) .
$$


Note that as in (1) we have

$$
\begin{aligned}
\left(\sum_{n=1}^{\infty} \sum_{l \in \mathbb{A}_{n}}\left(\left(\frac{e}{R}\right)^{n / p^{\prime}}\left(\begin{array}{c}
n \\
l
\end{array}\right)^{1 / p^{\prime}}|\alpha|^{l}\right)^{p^{\prime}}\right)^{1 / p^{\prime}} & =\left(\sum_{n=1}^{\infty}\left(\frac{e}{R}\right)^{n} \sum_{l \in \mathbb{A}_{n}}\left(\begin{array}{c}
n \\
l
\end{array}\right)\left|\alpha^{p^{\prime}}\right|^{l}\right)^{1 / p^{\prime}} \\
& \leq\left(\sum_{n=1}^{\infty}\left(\frac{e}{R}\right)^{n} \cdot 1\right)^{1 / p^{\prime}}=\left(\frac{e}{R-e}\right)^{1 / p^{\prime}} .
\end{aligned}
$$

The polarization formula gives $\left\|\stackrel{\vee}{P_{n}}\right\| \leq e^{n}\left\|P_{n}\right\|$ for every $n \in \mathbb{N}$ (see e.g. [9, Proposition 1.8]). And by (2) we obtain

$$
\begin{aligned}
\left(\sum_{n=1}^{\infty} \sum_{l \in \mathbb{A}_{n}}\left(\left(\begin{array}{c}
n \\
l
\end{array}\right)^{1 / p}\left(\frac{R}{e}\right)^{n / p^{\prime}}\left\|\stackrel{\vee}{P}_{n}\left(x^{l}\right)\right\|\right)^{p}\right)^{1 / p} \\
=\left(\sum_{n=1}^{\infty} \sum_{l \in \mathbb{A}_{n}}\left(\begin{array}{c}
n \\
l
\end{array}\right)\left(\left(\frac{R}{e}\right)^{p / p^{\prime}}\right)^{n}\left\|\stackrel{\vee}{P_{n}}\left(x^{l}\right)\right\|^{p}\right)^{1 / p} \\
\leq\left(\sum_{n=1}^{\infty}\left(\left(\frac{R}{e}\right)^{p / p^{\prime}}\right)^{n}\left\|\stackrel{\vee}{P}_{n}\right\|^{p}\left(\sum_{k=1}^{\infty}\left\|x_{k}\right\|^{p}\right)^{n}\right)^{1 / p} \\
\leq\left(\sum_{n=1}^{\infty}\left(\left(\frac{R}{e}\right)^{p / p^{\prime}}\right)^{n}\left(e^{n}\left\|P_{n}\right\|\right)^{p}\left(\sum_{k=1}^{\infty}\left\|x_{k}\right\|^{p}\right)^{n}\right)^{1 / p} \\
=\left(\sum_{n=1}^{\infty} \frac{1}{(1+\epsilon)^{n}}\left\|P_{n}\right\|^{p}\left((1+\epsilon)^{1 / p}\left(\frac{R}{e}\right)^{1 / p^{\prime}} e\left(\sum_{k=1}^{\infty}\left\|x_{k}\right\|^{p}\right)^{1 / p}\right) B_{X}\right)^{1 / p} \\
\leq\left(\sum_{n=1}^{\infty} \frac{1}{(1+\epsilon)^{n}}\left\|P_{n}\right\|_{s B_{X}}^{p}\right)^{1 / p} \leq\left(\sum_{n=1}^{\infty} \frac{1}{(1+\epsilon)^{n}}\|f\|_{s B_{X}}^{p}\right)^{1 / p} \\
=\|f\|_{s B_{X}}\left(\sum_{n=1}^{\infty} \frac{1}{(1+\epsilon)^{n}}\right)^{1 / p}=\frac{1}{\varepsilon^{\frac{1}{p}}}\|f\|_{s B_{X}}<\infty,
\end{aligned}
$$

where $\left\|P_{n}\right\|_{s B_{X}} \leq\|f\|_{s B_{X}}$ holds because of Cauchy's inequalities. Now

$$
f(x)=\frac{1}{2} y_{0}+\sum_{n=1}^{\infty} \sum_{l \in \mathbb{A}_{n}} a_{n, l} y_{n, l}
$$

where $y_{0}=2 f(0)$,

$$
a_{n, l}=\frac{1}{2}\left(\frac{R-e}{e}\right)^{1 / p^{\prime}}\left(\frac{e}{R}\right)^{n / p^{\prime}}\left(\begin{array}{c}
n \\
l
\end{array}\right)^{1 / p^{\prime}}|\alpha|^{l}
$$

and

$$
y_{n, l}=2\left(\frac{e}{R-e}\right)^{1 / p^{\prime}}\left(\begin{array}{l}
n \\
l
\end{array}\right)^{1 / p}\left(\frac{R}{e}\right)^{n / p^{\prime}} \stackrel{\vee}{P_{n}}\left(x^{l}\right),
$$

for $l \in \mathbb{A}_{n}$ and $n=1,2, \ldots$. Since

$$
\left(\left(\frac{1}{2}\right)^{p^{\prime}}+\sum_{n=1}^{\infty} \sum_{l \in \mathbb{A}_{n}}\left|a_{n, l}\right|^{p^{\prime}}\right)^{1 / p^{\prime}} \leq \frac{1}{2}+\frac{1}{2}=1
$$


and

$$
\left(\left\|y_{0}\right\|+\sum_{n=1}^{\infty} \sum_{l \in \mathbb{A}_{n}}\left\|y_{n, l}\right\|^{p}\right)^{1 / p} \leq 2\|f\|_{s B_{X}}+2\left(\frac{e}{R-e}\right)^{1 / p^{\prime}} \frac{1}{\varepsilon^{\frac{1}{p}}}\|f\|_{s B_{X}}<\infty,
$$

the proof is complete.

Theorem 3.5. Let $X$ and $Y$ be Banach spaces, and let $U$ be a balanced open subset of $X$. If $f \in \mathcal{H}(U ; Y)$ and

$$
K:=\left\{\sum_{k=1}^{\infty} \alpha_{k} x_{k}:\left(\alpha_{k}\right)_{k=1}^{\infty} \in \bar{B}_{\ell_{p^{\prime}}}\right\} \subset U
$$

is the fundamental $p$-compact set generated by the sequence $\left(x_{k}\right)_{k=1}^{\infty} \in \ell_{p}(X)$, then $f(K)$ is a p-compact subset of $Y$.

Proof. Again, we will provide a proof for the case $p>1$, the case $p=1$ being similar.

Let us take $R>1$ and $s>0$ such that $R^{2} K+s B_{X} \subset U$ and $\|f\|_{R^{2} K+s B_{X}}<+\infty$. Let $k_{0} \in \mathbb{N}$ such that $\left(\sum_{k=k_{0}}^{\infty}\left\|x_{k}\right\|^{p}\right)^{\frac{1}{p}}<\frac{s}{e}$.

Let $\eta: R^{2} B_{\ell_{p^{\prime}}^{k_{0}}} \longrightarrow X$ be defined by $\eta\left(\lambda_{1}, \ldots, \lambda_{k_{0}}\right)=\sum_{k=1}^{k_{0}} \lambda_{k} x_{k}$ for every element $\left(\lambda_{1}, \ldots, \lambda_{k_{0}}\right) \in R^{2} B_{\ell_{p^{\prime}}^{k_{0}}}$. For fixed $x \in X$ so that $\eta\left(R^{2} B_{\ell_{p^{\prime}}^{k_{0}}}\right)+x \subset U$ we define $g_{x}: R^{2} B_{\ell_{p^{\prime}}^{k_{0}}} \longrightarrow Y$ by $g_{x}(\lambda)=f(\eta(\lambda)+x)$ for every $\lambda \in R^{2} B_{\ell_{p^{\prime}}^{k_{0}}}$. By the Cauchy integral formula we have $g_{x}(\lambda)=\sum_{\alpha \in \mathbb{N}_{0}^{k_{0}}} c_{\alpha}(x) \lambda^{\alpha}$ with

$$
c_{\alpha}(x)=\frac{1}{(2 \pi i)^{k_{0}}} \int_{\left|\lambda_{1}\right|=r_{1}} \cdots \int_{\left|\lambda_{k_{0}}\right|=r_{k_{0}}} \frac{g_{x}(\lambda)}{\lambda^{\alpha+1}} d \lambda_{1} \ldots d \lambda_{k_{0}},
$$

for $r=\left(r_{1}, \ldots, r_{k_{0}}\right), r_{j}>0, j=1, \ldots, k_{0},\|r\|_{p^{\prime}}<R^{2}$, where $\mathbf{1}=(1, \ldots, 1)$. Therefore

$$
\left\|c_{\alpha}(x)\right\| \leq \frac{1}{r^{\alpha}} \sup _{|\lambda|=r}\|f(\eta(\lambda)+x)\| .
$$

Clearly, if $K_{1}=p$-co $\left\{x_{1}, \ldots, x_{k_{0}}\right\}$, then $\eta\left(t \bar{B}_{\ell_{p^{\prime}}^{k_{0}}}\right)=t K_{1}$ for every $0<t<R^{2}$. From [9, Lemma 1.38] we know that for $\alpha \in \mathbb{N}_{0}^{k_{0}}$

$$
\sup _{z \in \ell_{p^{\prime}}^{k_{0}}}\left|z^{\alpha}\right|=\left(\frac{\alpha^{\alpha}}{|\alpha|^{|\alpha|}}\right)^{\frac{1}{p^{\prime}}}
$$

and this supremum is attained at

$$
z_{0, \alpha}=\left(\left(\frac{\alpha_{1}}{|\alpha|}\right)^{\frac{1}{p^{\prime}}}, \ldots,\left(\frac{\alpha_{k_{0}}}{|\alpha|}\right)^{\frac{1}{p^{\prime}}}\right) \in S_{\ell_{p^{\prime}}^{k_{0}}} .
$$

By taking $r=z_{0, \alpha}$ in (5) we get

$$
\left\|c_{\alpha}\right\|_{s B_{X}}\left(R^{2} z_{0, \alpha}\right)^{\alpha} \leq\|f\|_{R^{2} K_{1}+s B_{X}}<+\infty
$$

for every $\alpha \in \mathbb{N}_{0}^{k_{0}}$. In other words every function $\left(R z_{0, \alpha}\right)^{\alpha} c_{\alpha}$ is holomorphic and bounded on $R^{2} K_{1}+s B_{X}$. Hence, we can apply Lemma 3.4 (for $\left(\sum_{k=k_{0}}^{\infty}\left\|x_{k}\right\|^{p}\right)^{\frac{1}{p}}<$ $\frac{s}{e}$ and all holomorphic and bounded functions on $\left.s B_{X}\right)$ to obtain a constant $M>0$ 
such that for any given function $\left(R z_{0, \alpha}\right)^{\alpha} c_{\alpha}$, there exists a sequence $\left(y_{j, \alpha}\right)_{j=1}^{\infty} \in$ $\ell_{p}(Y)$ satisfying

$$
\left(R z_{0, \alpha}\right)^{\alpha} c_{\alpha}\left(p-c o\left\{x_{k_{0}+1}, x_{k_{0}+2}, \ldots\right\}\right) \subset p-c o\left\{\left(y_{j, \alpha}\right)_{j=1}^{\infty}\right\}
$$

and

(ii) $\quad\left\|\left(y_{j, \alpha}\right)_{j=1}^{\infty}\right\|_{p} \leq M\left\|\left(R z_{0, \alpha}\right)^{\alpha} c_{\alpha}\right\|_{s B_{X}} \leq \frac{M}{R^{\alpha}}\|f\|_{R^{2} K+s B_{X}} \quad$ by $\quad$ (6) for every $\alpha \in \mathbb{N}_{0}^{k_{0}}$.

Observe that we have

$$
\begin{aligned}
& \left(\sum_{\alpha \in \mathbb{N}_{0}^{k_{0}}} \sum_{j=1}^{\infty}\left\|y_{j, \alpha}\right\|^{p}\right)^{\frac{1}{p}} \leq \sum_{\alpha \in \mathbb{N}_{0}^{k_{0}}}\left(\sum_{j=1}^{\infty}\left\|y_{j, \alpha}\right\|^{p}\right)^{\frac{1}{p}}=\sum_{\alpha \in \mathbb{N}_{0}^{k_{0}}}\left\|\left(y_{j, \alpha}\right)_{j=1}^{\infty}\right\|_{p} \\
\leq & \sum_{\alpha \in \mathbb{N}_{0}^{k_{0}}} \frac{M}{R^{\alpha}}\|f\|_{R^{2} K+s B_{X}}=\left(\sum_{h=0}^{\infty} \frac{1}{R^{h}}\right)^{k_{0}} M\|f\|_{R^{2} K+s B_{X}} \\
= & \left(\frac{R}{R-1}\right)^{k_{0}} M\|f\|_{R^{2} K+s B_{X}}<+\infty .
\end{aligned}
$$

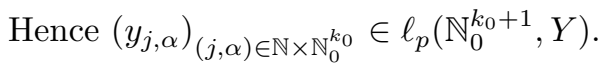

Consider now $\left(\lambda_{k}\right)_{k=1}^{\infty} \in \bar{B}_{\ell_{p^{\prime}}}$. We write $\sum_{k=1}^{\infty} \lambda_{k} x_{k}=\sum_{k=1}^{k_{0}} \lambda_{k} x_{k}+x$, where $x=\sum_{k=k_{0}+1}^{\infty} \lambda_{k} x_{k}$. So

$$
f\left(\sum_{k=1}^{\infty} \lambda_{k} x_{k}\right)=g_{x}(\lambda)=\sum_{\alpha \in \mathbb{N}_{0}^{k_{0}}} c_{\alpha}(x) \lambda^{\alpha}=\sum_{\alpha \in \mathbb{N}_{0}^{k_{0}}}\left(R z_{0, \alpha}\right)^{\alpha} c_{\alpha}(x)\left(\frac{\lambda}{R z_{0, \alpha}}\right)^{\alpha},
$$

where $\lambda=\left(\lambda_{1}, \ldots, \lambda_{k_{0}}\right) \in \bar{B}_{\ell_{p^{\prime}}^{k_{0}}}$.

By $(i)$, for every $\alpha \in \mathbb{N}_{0}^{k_{0}}$, there exists $\left(\beta_{j, \alpha}\right) \in \bar{B}_{\ell_{p^{\prime}}}$ such that $\left(R z_{0, \alpha}\right)^{\alpha} c_{\alpha}(x)=$ $\sum_{j=1}^{\infty} \beta_{j, \alpha} y_{j, \alpha}$. Thus

$$
f\left(\sum_{k=1}^{\infty} \lambda_{k} x_{k}\right)=\sum_{\alpha \in \mathbb{N}_{0}^{k_{0}}}\left(\sum_{j=1}^{\infty} \beta_{j, \alpha} \frac{\left(\frac{R}{R-1}\right)^{\frac{k_{0}}{p^{\prime}}}}{\left(\frac{R}{R-1}\right)^{\frac{k_{0}}{p^{\prime}}}} y_{j, \alpha}\right) \frac{1}{R^{\alpha}}\left(\frac{\lambda}{z_{0, \alpha}}\right)^{\alpha} .
$$

But $|\lambda|^{\alpha} \leq\left|z_{0, \alpha}\right|^{\alpha}$, so $\frac{|\lambda|^{\alpha}}{\left|z_{0, \alpha}\right|^{\alpha}} \leq 1$. Thus

$$
\begin{gathered}
\sum_{\alpha \in \mathbb{N}_{0}^{k_{0}}} \frac{1}{\left(\frac{R}{R-1}\right)^{k_{0}}} \sum_{j=1}^{\infty}\left|\beta_{j, \alpha}\right|^{p^{\prime}}\left(\frac{1}{R^{\alpha}}\right)^{p^{\prime}}\left(\frac{|\lambda|}{\left|z_{0, \alpha}\right|}\right)^{\alpha p^{\prime}} \\
\leq \frac{1}{\left(\frac{R}{R-1}\right)^{k_{0}}} \sum_{\alpha \in \mathbb{N}_{0}^{k_{0}}} \sum_{j=1}^{\infty}\left|\beta_{j, \alpha}\right|^{p^{\prime}}\left(\frac{1}{R^{\alpha}}\right)^{p^{\prime}} \leq \frac{1}{\left(\frac{R}{R-1}\right)^{k_{0}}} \sum_{\alpha \in \mathbb{N}_{0}^{k_{0}}}\left(\frac{1}{R^{\alpha}}\right)^{p^{\prime}} \\
\leq \frac{1}{\left(\frac{R}{R-1}\right)^{k_{0}}} \sum_{\alpha \in \mathbb{N}_{0}^{k_{0}}} \frac{1}{R^{\alpha}}=\frac{1}{\left(\frac{R}{R-1}\right)^{k_{0}}}\left(\sum_{h=0}^{\infty} \frac{1}{R^{h}}\right)^{k_{0}}=1, \\
\text { i.e., }\left(\frac{1}{\left(\frac{R}{R-1}\right)^{\frac{k_{0}}{p^{\prime}}}} \beta_{j, \alpha} \frac{1}{R^{\alpha}}\left(\frac{\lambda}{z_{0, \alpha}}\right)^{\alpha}\right)_{(j, \alpha) \in \mathbb{N} \times \mathbb{N}_{0}^{k_{0}}} \in \bar{B}_{\ell_{p^{\prime}}} .
\end{gathered}
$$


Hence by (7)

$$
f(K) \subset p-\operatorname{co}\left\{\left(\left(\frac{R}{R-1}\right)^{\frac{k_{0}}{p^{\prime}}} y_{j, \alpha}\right)_{(j, \alpha) \in \mathbb{N} \times \mathbb{N}_{0}^{k_{0}}}\right\}
$$

and $\left(\left(\frac{R}{R-1}\right)^{\frac{k_{0}}{p^{\prime}}} y_{j, \alpha}\right)_{(j, \alpha) \in \mathbb{N} \times \mathbb{N}_{0}^{k_{0}}} \in \ell_{p}(Y)$, i.e., $f(K)$ is $p$-compact.

Corollary 3.6. Let $X$ and $Y$ be Banach spaces and let $f \in \mathcal{H}(X ; Y)$. If $K \subset X$ is a p-compact set, then the set $f(K) \subset Y$ is a p-compact set.

Remark 3.7. Let $U$ be an open balanced subset of a complex Banach space $X$ (or even $U$ an open balanced and convex set). We do not know if given a $p$-compact set $K$ contained in $U$, there necessarily exists a sequence $\left(x_{k}\right)_{k=1}^{\infty}$ contained in $\ell_{p}(X) \cap U$ such that $K \subset p$-co $\left\{\left(x_{k}\right)_{k=1}^{\infty}\right\}$. If this were the case, then by Theorem 3.5 the image of any $p$-compact set under any holomorphic function on $U$ would again be $p$-compact.

\section{LOCALIZATION OF $p$-COMPACT SETS OF SYMMETRIC TENSOR PRODUCTS OF A BANACH SPACE}

We now give a result related to the localization of $p$-compact sets of symmetric tensor products of a Banach space. Let $\widehat{\otimes_{s, m}, \pi} X$ denote the complete $m$-fold symmetric tensor product of $X$ with the projective norm topology $\pi$, and let $q_{m}$ : $X \longrightarrow \widehat{\otimes_{s, m, \pi}} X$ be the $m$-homogeneous polynomial defined by $q_{m}(x):=x \otimes \cdots \otimes x$, for every $x \in X$. In his thesis R. Ryan [14] showed that given a compact subset $K$ of $\widehat{\otimes_{s, m, \pi}} X$, there is a sequence $\left(z_{l}\right)_{l=1}^{\infty} \in c_{0}(X)$ such that $K \subset \overline{\Gamma\left\{\left(q_{m}\left(z_{l}\right)\right)_{l=1}^{\infty}\right\}}$ (see [14, Proposition 2.5]), which is related to the localization of compact sets in $\widehat{\otimes_{s, m, \pi}} X$. In the $p$-compact case we have the following similar result which extends Ryan's work beyond the $p=\infty$ case.

Proposition 4.1. Let $X$ be a Banach space and let $m \in \mathbb{N}$. Then, given a $p$ compact subset $K$ of $\widehat{\otimes_{s, m, \pi}} X$, there is a sequence $\left(z_{l}\right)_{l=1}^{\infty} \in \ell_{m p}(X)$ such that $K \subset p$-co $\left\{\left(q_{m}\left(z_{l}\right)\right)_{l=1}^{\infty}\right\}$.

Proof. (a) Let $K \subset \widehat{\otimes_{s, m, \pi}} X$ be a $p$-compact subset. Then, there is a sequence $\left(\tau_{k}\right)_{k=1}^{\infty} \in \ell_{p}\left(\widehat{\otimes_{s, m, \pi}} X\right)$ such that $K \subset\left\{\sum_{k=1}^{\infty} \lambda_{k} \tau_{k}:\left(\lambda_{k}\right)_{k=1}^{\infty} \in \bar{B}_{\ell_{p^{\prime}}}\right\}$. Let $\tau \in K$ be any element. Then, there is a sequence $\left(\lambda_{k}\right)_{k=1}^{\infty} \in \bar{B}_{\ell_{p^{\prime}}}$ such that $\tau=\sum_{k=1}^{\infty} \lambda_{k} \tau_{k}$. By [14, Proposition 2.5], for each $\tau_{k} \in \widetilde{\otimes_{s, m, \pi}} X$ we can find a representation of the form

$$
\tau_{k}=\sum_{i=1}^{\infty} \lambda_{k, i} \underbrace{x_{k, i} \otimes \cdots \otimes x_{k, i}}_{m \text { times }}, \quad x_{k, i} \in X, \lambda_{k, i} \in \mathbb{C},
$$

with $\left\|x_{k, i}\right\|=1$ for every $i \in \mathbb{N}$, such that

$$
\mu_{k}:=\sum_{i=1}^{\infty}\left|\lambda_{k, i}\right| \leq \pi\left(\tau_{k}\right)+\frac{1}{2^{k}} .
$$


(See, also, [15, Proposition 2.8] and the formulas given for $\pi$ in [15, page 22].) Now, for each $k$ using the corresponding representation and writing

$$
\begin{aligned}
\tau & =\sum_{k=1}^{\infty} \lambda_{k} \sum_{i=1}^{\infty} \frac{\lambda_{k, i}}{\mu_{k}} \mu_{k}^{1 / m} x_{k, i} \otimes \cdots \otimes \mu_{k}^{1 / m} x_{k, i} \\
& =\sum_{k=1}^{\infty} \sum_{i=1}^{\infty} \lambda_{k}\left(\frac{\lambda_{k, i}}{\mu_{k}}\right)^{1 / p^{\prime}}\left(\frac{\lambda_{k, i}}{\mu_{k}}\right)^{1 /(m p)} \mu_{k}^{1 / m} x_{k, i} \otimes \cdots \otimes\left(\frac{\lambda_{k, i}}{\mu_{k}}\right)^{1 /(m p)} \mu_{k}^{1 / m} x_{k, i}
\end{aligned}
$$

we see that

$$
\begin{aligned}
\sum_{k=1}^{\infty} \sum_{i=1}^{\infty}\left|\lambda_{k}\left(\frac{\lambda_{k, i}}{\mu_{k}}\right)^{1 / p^{\prime}}\right|^{p^{\prime}} & =\sum_{k=1}^{\infty} \sum_{i=1}^{\infty}\left|\lambda_{k}\right|^{p^{\prime}}\left|\frac{\lambda_{k, i}}{\mu_{k}}\right| \\
& \leq \sum_{k=1}^{\infty}\left|\lambda_{k}\right|^{p^{\prime}} \sum_{i=1}^{\infty}\left|\frac{\lambda_{k, i}}{\mu_{k}}\right| \leq \sum_{k=1}^{\infty}\left|\lambda_{k}\right|^{p^{\prime}} \leq 1
\end{aligned}
$$

and that

$$
\begin{aligned}
\sum_{k=1}^{\infty} \sum_{i=1}^{\infty} \|\left(\frac{\lambda_{k, i}}{\mu_{k}}\right)^{1 /(m p)} & \mu_{k}^{1 / m} x_{k, i}\left\|^{m p} \leq \sum_{k=1}^{\infty} \sum_{i=1}^{\infty}\left|\frac{\lambda_{k, i}}{\mu_{k}}\right| \mu_{k}^{p}\right\| x_{k, i} \|^{m p} \\
& \leq \sum_{k=1}^{\infty} \mu_{k}^{p} \sum_{i=1}^{\infty}\left|\frac{\lambda_{k, i}}{\mu_{k}}\right| \leq \sum_{k=1}^{\infty} \mu_{k}^{p} \leq \sum_{k=1}^{\infty}\left(\pi\left(\tau_{k}\right)+\frac{1}{2^{k}}\right)^{p}<\infty
\end{aligned}
$$

Therefore,

$$
\left(\lambda_{k}\left(\frac{\lambda_{k, i}}{\mu_{k}}\right)^{1 / p^{\prime}}\right)_{(k, i) \in \mathbb{N} \times \mathbb{N}} \in \bar{B}_{\ell_{p^{\prime}}},\left(\left(\frac{\lambda_{k, i}}{\mu_{k}}\right)^{1 /(m p)} \mu_{k}^{1 / m} x_{k, i}\right)_{(k, i) \in \mathbb{N} \times \mathbb{N}} \in \ell_{m p}(X),
$$

and also

$$
\left(\left(\frac{\lambda_{k, i}}{\mu_{k}}\right)^{1 /(m p)} \mu_{k}^{1 / m} x_{k, i} \otimes \cdots \otimes\left(\frac{\lambda_{k, i}}{\mu_{k}}\right)^{1 /(m p)} \mu_{k}^{1 / m} x_{k, i}\right)_{(k, i) \in \mathbb{N} \times \mathbb{N}} \in l_{p}\left(\widehat{\bigotimes_{s, m, \pi}} X\right) .
$$

Therefore, since the double series considered above are all absolutely convergent, by a reordering we obtain sequences

$$
\begin{gathered}
\left(\gamma_{l}\right)_{l=1}^{\infty}:=\left(\lambda_{k}\left(\frac{\lambda_{k, i}}{\mu_{k}}\right)^{1 / p^{\prime}}\right)_{(k, i) \in \mathbb{N} \times \mathbb{N}} \in \bar{B}_{\ell_{p^{\prime}}}, \\
\left(z_{l}\right)_{l=1}^{\infty}:=\left(\left(\frac{\lambda_{k, i}}{\mu_{k}}\right)^{1 /(m p)} \mu_{k}^{1 / m} x_{k, i}\right)_{(k, i) \in \mathbb{N} \times \mathbb{N}} \in \ell_{m p}(X) .
\end{gathered}
$$

Thus we have shown the existence of a sequence $\left(z_{l}\right)_{l=1}^{\infty} \in \ell_{m p}(X)$ such that

$$
K \subset p-c o\left\{\left(z_{l} \otimes \cdots \otimes z_{l}\right)_{l=1}^{\infty}\right\}=p-c o\left\{\left(q_{m}\left(z_{l}\right)\right)_{l=1}^{\infty}\right\},
$$

as we desired.

In the study of the images of $p$-compact sets by polynomials, note that the image of a $p$-summable sequence by an $m$-homogeneous polynomial is $p / m$-summable. This fact naturally gives rise to the following question: Must the image of a $p$ compact set under an $m$-homogeneous polynomial be $p / m$-compact? We will show 
below that this is not always the case. More precisely, we shall give an example of an $m$-homogeneous polynomial having the following properties:

(i) The image of a certain $p$-summable sequence by this polynomial is zero, although

(ii) The image of a $p$-compact set generated by a certain sequence under this polynomial is not even $q$-compact for any $1 \leq q<p$.

Example 4.2. For every $m \in \mathbb{N}$, there exist an $m$-homogeneous continuous polynomial $P: \ell_{p} \longrightarrow \ell_{p}$ and a $p$-compact subset $K$ of $\ell_{p}$ such that $P(K)$ is (p-compact but) not $q$-compact for any $1 \leq q<p$.

Proof. Let us define an $m$-homogeneous polynomial $P: \ell_{p} \longrightarrow \ell_{p}$ by

$$
P(x):=x_{1}^{m-1} \sum_{j=1}^{\infty} x_{2 j} e_{2 j}, \quad x=\left(x_{n}\right)_{n=1}^{\infty} \in \ell_{p},
$$

where $\left(e_{h}\right)_{h=1}^{\infty}$ is the canonical basis of $\ell_{p}$. Note that, for every $h \in \mathbb{N}, P\left(e_{h}\right)=0$ and

$$
P\left(e_{1}+a_{h} e_{2 h}\right)=a_{h} e_{2 h}, \quad \text { for every } h \in \mathbb{N} \text { and } a_{h} \in \mathbb{C} .
$$

At this point note that the image of a sequence $\left\{b_{n} e_{n}\right\}_{n=1}^{\infty}$ with $\left(b_{n}\right)_{n=1}^{\infty} \in \ell_{p}$, which generates a $p$-compact set, under $P$ is zero. But now we will see that the image of a $p$-compact set generated by a certain $p$-summable sequence can have a quite different nature.

If for any $p \geq 1$ we let $a_{h}:=\frac{1}{\sqrt[p]{h}(\log h)^{2}}, h=3,4, \ldots$, then $\left(a_{h}\right)_{h=3}^{\infty} \in \ell_{p}$, whereas $\left(a_{h}\right)_{h=3}^{\infty} \notin \ell_{q}$ for every $0<q<p$. Indeed, if $\sum_{h=1}^{\infty} a_{h}^{q}<\infty$, then it would follow that $\lim _{h \longrightarrow \infty} h a_{h}^{q}=0$. However $\lim _{h \longrightarrow \infty} \frac{h}{h^{q / p}(\log h)^{2 q}}=\infty$. Now, as the sequence $\left(2 e_{1}, \frac{2}{\sqrt[p]{h}(\log h)^{2}} e_{2 h}: h=3,4, \ldots\right)$ belongs to $\ell_{p}\left(\ell_{p}\right)$, by definition

$$
K:=p-c o\left\{2 e_{1}, \frac{2}{\sqrt[p]{h}(\log h)^{2}} e_{2 h}: h=3,4, \ldots\right\}
$$

is $p$-compact. By Lemma 3.2 we know that $P(K)$ is $p$-compact. We claim that $P(K)$ is not $q$-compact for any $1 \leq q<p$. Indeed, the sequence $\left(e_{1}+\frac{2}{\sqrt[p]{h}(\log h)^{2}} e_{2 h}\right.$ : $h=3,4, \ldots)$ is included in $K$ and by $(*)$ we have that

$$
P\left(e_{1}+\frac{1}{\sqrt[p]{h}(\log h)^{2}} e_{2 h}\right)=\frac{1}{\sqrt[p]{h}(\log h)^{2}} e_{2 h}, \text { for every } h=3,4, \ldots
$$

If we define a sequence $\left(\alpha_{n}\right)_{n=1}^{\infty}$ by

$$
\alpha_{n}= \begin{cases}0 & \text { if } n \text { is odd } \\ \frac{1}{\sqrt[p]{\frac{n+4}{2}}\left(\log \frac{n+4}{2}\right)^{2}} & \text { if } n \text { is even, }\end{cases}
$$

we have that $\left(\alpha_{n}\right)_{n=1}^{\infty} \in \ell_{p} \backslash \ell_{q}$ for every $q<p$, so that by the proof of [1, Example 1] we see that the image $P\left(\left(e_{1}+\frac{1}{\sqrt[p]{h}(\log h)^{2}} e_{2 h}\right)_{h=3}^{\infty}\right)=\left\{\left(\alpha_{n} e_{n}\right)_{n=1}^{\infty}\right\}$ is not $q$ compact for any $q<p$. Hence, since $P(K) \supset P\left(\left(e_{1}+\frac{1}{\sqrt[p]{h}(\log h)^{2}} e_{2 h}\right)_{h=3}^{\infty}\right)$, we conclude that $P(K)$ cannot be $q$-compact for any $q<p$.

Now we consider the question of the pulling back of $p$-compact subsets of the image. That is, if $P: X \longrightarrow Y$ is an $m$-homogeneous polynomial and $K \subset P(X)$ is 
a $p$-compact set, does there exist a $p$-compact subset $L \subset X$ such that $K \subset P(L)$ ? The following shows that the answer in general is negative.

Proposition 4.3. Let $m \in \mathbb{N}, m \geq 2$, and let $q_{m}: \ell_{p} \longrightarrow \widehat{\mathbb{Q}_{s, m}} \ell_{p}$ be defined by $q_{m}(x):=\underbrace{x \otimes \cdots \otimes x}_{m \text { times }}, x \in \ell_{p}$. There is a p-compact subset $K$ of $\overline{\otimes_{s, m, \pi}} \ell_{p}$ such that a set $L \subset \ell_{p}$ is $r$-compact and $K \subset q_{m}(L)$ if and only if $r \geq p m$.

Proof. Let us consider a sequence of strictly positive numbers $\left(a_{h}\right)_{h=1}^{\infty} \in \ell_{p} \backslash \ell_{q}$, where $0<q<p<\infty$, and take

$$
K:=\{a_{h} \underbrace{e_{h} \otimes \cdots \otimes e_{h}}_{m \text { times }}: h \in \mathbb{N}\} \cup\{0\},
$$

where $\left(e_{h}\right)_{h=1}^{\infty}$ is the canonical basis of $\ell_{p}$. Since $\pi\left(a_{h} e_{h} \otimes \cdots \otimes e_{h}\right)=a_{h}, K$ is p-compact in $\widehat{\otimes_{s, m, \pi}} \ell_{p}$.

We claim that if a subset $L \subset \ell_{p}$ is $r$-compact and $K \subset q_{m}(L)$, then $r \geq p m$. To see this, note that $q_{m}^{-1}\left(a_{h} e_{h} \otimes \cdots \otimes e_{h}\right)=\left\{\lambda \sqrt[m]{a_{h}} e_{h}: \lambda \in \mathbb{C}, \lambda^{m}=1\right\}$. Hence, since for every $h \in \mathbb{N}, q_{m}\left(\sqrt[m]{a_{h}} e_{h}\right)=a_{h} e_{h} \otimes \cdots \otimes e_{h}$, there is a $\lambda_{h} \in \mathbb{C}$ with $\lambda_{h}^{m}=1$ such that $\lambda_{h} \sqrt[m]{a_{h}} e_{h} \in L$. Since we can take $L$ to be balanced, it follows that $\sqrt[m]{a_{h}} e_{h} \in L$, for every $h \in \mathbb{N}$. But, since $\left(\sqrt[m]{a_{h}}\right)_{h=1}^{\infty} \in l_{p m} \backslash l_{k}, 0<k<p m$, by a modification of the proof of [1, Example 1] we see that $\left(\sqrt[m]{a_{h}} e_{h}\right)_{h=1}^{\infty} \cup\{0\}$ cannot be $k$-compact for any $0<k<p m$, so that the set $L$ cannot be $k$-compact for $0<k<p m$. Therefore $r \geq p m$.

Now, the rest of the proof is a consequence of Proposition 4.1

We end this section with the following observations.

Remarks 4.4. (a) Let $m \in \mathbb{N}$ with $m \leq p$, and let $K$ be a subset of $\widehat{\otimes_{s, m, \pi}} X$. If there is a sequence $\left(a_{n}\right)_{n=1}^{\infty} \in \ell_{p}(X)$ such that $K \subset r-c o\left\{\left(a_{n} \otimes \cdots \otimes a_{n}\right)_{n=1}^{\infty}\right\}$ for any $r \geq 1$, then $K$ is a 1 -compact subset of $\widehat{\otimes_{s, m, \pi}} X$.

(b) Let $K$ be a subset of $\widehat{\otimes_{s, m, \pi}} X$. If there is a sequence $\left(a_{n}\right)_{n=1}^{\infty} \in \ell_{p}(X)$, $p \geq 1$, such that $K \subset r-c o\left\{\left(a_{n} \otimes \cdots \otimes a_{n}\right)_{n=1}^{\infty}\right\}$ for any $r \geq p$, then $K$ is a $p$-compact subset of $\widehat{\bigotimes_{s, m, \pi}} X$.

\section{ON $p$-COMPACT Linear OPERATORS BETWEen $\ell_{p}$ SPACES}

The definition of $p$-compact sets leads naturally to the notion of $p$-compact operators which is introduced in [16].

Definition 5.1. An operator $T \in \mathcal{L}(X ; Y)$ is called $p$-compact if there exists a sequence $\left(y_{n}\right)_{n=1}^{\infty} \in \ell_{p}(Y)\left(\left(y_{n}\right)_{n=1}^{\infty} \in c_{0}(Y)\right.$ if $\left.p=\infty\right)$ such that

$$
T\left(\bar{B}_{X}\right) \subset\left\{\sum_{n=1}^{\infty} \alpha_{n} y_{n}:\left(\alpha_{n}\right)_{n=1}^{\infty} \in \bar{B}_{\ell_{p^{\prime}}}\right\} .
$$

Let $\mathcal{K}_{p}(X ; Y)$ denote the subspace of all $p$-compact operators of $\mathcal{L}(X ; Y)$. Clearly $\mathcal{K}_{p}(X ; Y) \subset \mathcal{K}(X ; Y)$. Note also that for $1 \leq p<q \leq \infty$ we have $\mathcal{K}_{p}(X ; Y) \subset$ $\mathcal{K}_{q}(X ; Y)$ (see [16, Proposition 4.3]) and below we will see that this inclusion is in general strict.

On the other hand if $1<p<q<\infty$, by Pitt's Theorem we know that $\mathcal{L}\left(\ell_{q} ; \ell_{p}\right)=$ $\mathcal{K}\left(\ell_{q} ; \ell_{p}\right)$, that is, every continuous linear operator from $\ell_{q}$ into $\ell_{p}$ is compact. But 
the following proposition, which is a modification of [1, Example 1], shows that in the $p$-compact case there are linear continuous operators from $\ell_{q}$ to $\ell_{p}, 1 \leq p<q$, which are not $p$-compact. More precisely we have the following.

Proposition 5.2. There is a linear operator $T \in \mathcal{L}\left(\ell_{q} ; \ell_{p}\right)$ which is not $r$-compact for $1 \leq r \leq p$, if

(a) $1 \leq p \leq q \leq p q^{\prime}$, or

(b) $1<p \leq q \leq \min \left\{p^{2}, p p^{\prime}\right\}$.

Furthermore, in each case the corresponding operator is q-compact if, in addition, $p<q \leq q^{\prime}$.

Proof. We shall only prove the case $(a)$, the other case being similar.

First, note that if $1 \leq p=q$, then the identity operator from $\ell_{p}$ to $\ell_{p}$ is not compact, so that it is not $r$-compact for any $1 \leq r$.

Now, let $1 \leq p<q \leq p q^{\prime}$ and let $T: \ell_{q} \longrightarrow \ell_{p}$ be defined by

$$
T\left(\left(x_{n}\right)_{n=1}^{\infty}\right):=\left(a_{n} x_{n}\right)_{n=1}^{\infty}, \quad\left(x_{n}\right)_{n=1}^{\infty} \in \ell_{q},
$$

where $\left(a_{n}\right)_{n=1}^{\infty} \in \ell_{q} \backslash \ell_{p}$. As $p q \geq q$ we have that $\left(a_{n}\right)_{n=1}^{\infty} \in \ell_{p q}$, i.e. $\left(\left|a_{n}\right|^{p}\right)_{n=1}^{\infty} \in \ell_{q}$. On the other hand, by hypothesis, $p q^{\prime} \geq q$, thus $\left(x_{n}\right)_{n=1}^{\infty} \in \ell_{p q^{\prime}}$, i.e. $\left(\left|x_{n}\right|^{p}\right)_{n=1}^{\infty} \in \ell_{q^{\prime}}$. Hence, by Hölder's inequality, $\left(\left|a_{n} x_{n}\right|^{p}\right)_{n=1}^{\infty} \in \ell_{1}$. Therefore $T$ is well defined.

It is sufficient to prove the claim for $r=p$. Suppose, for a contradiction, that $T \in \mathcal{K}_{p}\left(\ell_{q} ; \ell_{p}\right)$. Then there is a sequence $\left(z_{k}\right)_{k=1}^{\infty} \in \ell_{p}\left(\ell_{p}\right)$, each $z_{k}=\sum_{l=1}^{\infty} z_{l}^{k} e_{l} \in$ $\ell_{p}$, such that

$$
T\left(\bar{B}_{\ell_{q}}\right) \subset p-c o\left\{\left(z_{k}\right)_{k=1}^{\infty}\right\}=\left\{\sum_{k=1}^{\infty} \alpha_{k} z_{k}:\left(\alpha_{k}\right)_{k=1}^{\infty} \in \bar{B}_{\ell_{p^{\prime}}}\right\},
$$

where $\left(e_{l}\right)_{l=1}^{\infty}$ is the canonical basis. Hence, in particular, we have that

$$
\left\{\left(T\left(e_{n}\right)\right)_{n=1}^{\infty}\right\}=\left\{\left(a_{n} e_{n}\right)_{n=1}^{\infty}\right\} \subset\left\{\sum_{k=1}^{\infty} \alpha_{k} z_{k}:\left(\alpha_{k}\right)_{k=1}^{\infty} \in \bar{B}_{\ell_{p^{\prime}}}\right\} .
$$

Here we actually show that this last inclusion cannot be true; that is, the set $\left\{\left(a_{n} e_{n}\right)_{n=1}^{\infty}\right\}$ cannot be $p$-compact (note that this set is $q$-compact since it belongs to $\left.\ell_{q}\left(\ell_{p}\right)\right)$. To see this, let us fix any $n \in \mathbb{N}$. Then there is a sequence $\left(\lambda_{k}^{n}\right)_{k=1}^{\infty} \in \bar{B}_{\ell_{p^{\prime}}}$ so that

$$
a_{n} e_{n}=\sum_{k=1}^{\infty} \lambda_{k}^{n} z_{k}=\sum_{k=1}^{\infty} \sum_{l=1}^{\infty} \lambda_{k}^{n} z_{l}^{k} e_{l}
$$

from which it follows that $a_{n}=\sum_{k=1}^{\infty} \lambda_{k}^{n} z_{n}^{k}$ and $\sum_{k=1}^{\infty} \lambda_{k}^{n} z_{l}^{k}=0$ if $l \neq n$. Now, by Hölder's inequality we get

$$
\begin{aligned}
\sum_{n=1}^{\infty}\left|a_{n}\right|^{p} & =\sum_{n=1}^{\infty}\left|\sum_{k=1}^{\infty} \lambda_{k}^{n} z_{n}^{k}\right|^{p} \\
& \leq \sum_{n=1}^{\infty}\left(\sum_{k=1}^{\infty}\left|\lambda_{k}^{n}\right|^{p^{\prime}}\right)^{p / p^{\prime}} \sum_{k=1}^{\infty}\left|z_{n}^{k}\right|^{p} \leq \sum_{n=1}^{\infty} \sum_{k=1}^{\infty}\left|z_{n}^{k}\right|^{p}<\infty
\end{aligned}
$$

which is a contradiction since $\left(a_{n}\right)_{n=1}^{\infty} \notin \ell_{p}$. Hence the set $\left\{\left(a_{n} e_{n}\right)_{n=1}^{\infty}\right\}$ is not $p$-compact, so $T$ is not a $p$-compact operator. Consequently, $T$ is not an $r$-compact operator for any $1 \leq r \leq p$.

Finally observe that $T$ is $q$-compact if, in addition, $q^{\prime} \geq q>p$. 
The above proposition can be seen as a partial complement to a recent result of J. M. Delgado and C. Piñeiro [6, Corollary 3.8]: (i) $\mathcal{L}\left(\ell_{r} ; \ell_{1}\right)=\mathcal{K}_{2}\left(\ell_{r} ; \ell_{1}\right)$ for every $r \geq 2$ and (ii) if $p>2$, then $\mathcal{L}\left(\ell_{r} ; \ell_{1}\right)=\mathcal{K}_{p}\left(\ell_{r} ; \ell_{1}\right)$ for every $r>p^{\prime}$. Actually, Proposition 5.2 shows, in particular, that $\mathcal{L}\left(\ell_{2} ; \ell_{1}\right) \neq \mathcal{K}_{1}\left(\ell_{2}, \ell_{1}\right)$, which is a case that is not covered by the results given in [6].

Also we note that, in [6. Proposition 3.10], Delgado and Piñeiro assert that if $1 \leq p, 1<q$ and $\mathcal{L}\left(X ; \ell_{q}\right)=\mathcal{K}_{p}\left(X ; \ell_{q}\right)$, then $X$ must be finite dimensional. In particular, this shows that $\mathcal{L}\left(c_{0} ; \ell_{q}\right) \neq \mathcal{K}_{p}\left(c_{0} ; \ell_{q}\right)$ when $1 \leq p, 1<q$. We do not know of any concrete example of a non-p-compact linear operator for the case $X=c_{0}$.

The sequence $\left(x_{n}\right)_{n=1}^{\infty}$ in the definition of $p$-compact set is not unique, so following [11] it is natural to consider

$$
\mathfrak{m}_{p}(K ; X):=\inf \left\{\left\|\left(x_{n}\right)_{n=1}^{\infty}\right\|_{p}: K \subset p-c o\left\{\left(x_{n}\right)_{n=1}^{\infty}\right\}\right\} .
$$

If $K \subset X$ is a $p$-compact set, then it follows from Hölder's inequality that $\|x\| \leq$ $\mathfrak{m}_{p}(K ; X)$, for every $x \in K$. Hence, as noted in [11, in this sense $\mathfrak{m}_{p}(K ; X)$ measures the size of $K$ as a $p$-compact subset of $X$.

In [8, Proposition 3.15] it is shown that the norm $k_{p}$ on $\mathcal{K}_{p}(X ; Y)$, defined by

$$
k_{p}(T):=\inf \left\{\left(\sum_{n=1}^{\infty}\left\|y_{n}\right\|^{p}\right)^{1 / p}:\left(y_{n}\right)_{n=1}^{\infty} \in \ell_{p}(Y) \text { and } T\left(\bar{B}_{X}\right) \subset p-c o\left\{\left(y_{n}\right)_{n=1}^{\infty}\right\}\right\},
$$

coincides with the ideal norm defined by Sinha and Karn in [16. It is easy to see that $\|T\| \leq k_{p}(T)$ for any $T \in \mathcal{K}_{p}(X ; Y)$. Note also that, according to the definitions, $\mathfrak{m}_{p}\left(T\left(\bar{B}_{X}\right) ; Y\right)=k_{p}(T)$. Below we will see that the size of $p$-compactness of the image of the unit ball can be arbitrarily large. That is, given any arbitrarily large positive number $R$, we can find a $p$-compact linear operator from $\ell_{q}$ to $\ell_{p}$, with norm $\leq 1$, such that its ideal norm is bigger than $R$.

Proposition 5.3. Given any number $R>0$ there is an operator $T \in \mathcal{K}_{p}\left(\ell_{q} ; \ell_{p}\right)$ with $\|T\| \leq 1$, but $k_{p}(T)>R$, where $1 \leq q \leq p<\infty$.

Proof. Let $1 \leq q \leq p<\infty$ and let $R>0$ be any number. Let us take any element $\left(a_{n}\right)_{n=1}^{\infty} \in \bar{B}_{\ell_{\infty}} \cap \ell_{p}$ such that $\left\|\left(a_{n}\right)_{n=1}^{\infty}\right\|_{p}>R$. Let $T: \ell_{q} \longrightarrow \ell_{p}$ be defined by

$$
T\left(\left(\beta_{n}\right)_{n=1}^{\infty}\right):=\left(a_{n} \beta_{n}\right)_{n=1}^{\infty}=\sum_{n=1}^{\infty} \beta_{n}\left(a_{n} e_{n}\right), \quad\left(\beta_{n}\right)_{n=1}^{\infty} \in \ell_{q} .
$$

Now, if we take $\left(\beta_{n}\right)_{n=1}^{\infty} \in \ell_{q}$ with $\left\|\left(\beta_{n}\right)_{n=1}^{\infty}\right\|_{q} \leq 1$, then it follows that

$$
\left\|T\left(\left(\beta_{n}\right)_{n=1}^{\infty}\right)\right\|_{p}=\left(\sum_{n=1}^{\infty}\left|a_{n} \beta_{n}\right|^{p}\right)^{1 / p} \leq\left(\sum_{n=1}^{\infty}\left|\beta_{n}\right|^{p}\right)^{1 / p} \leq\left(\sum_{n=1}^{\infty}\left|\beta_{n}\right|^{q}\right)^{1 / q} \leq 1
$$

which shows that $\|T\| \leq 1$. Note that $\left(a_{n} e_{n}\right)_{n=1}^{\infty} \in \ell_{p}\left(\ell_{p}\right)$ and that

$$
T\left(\bar{B}_{\ell_{q}}\right)=\left\{\sum_{n=1}^{\infty} \beta_{n}\left(a_{n} e_{n}\right):\left(\beta_{n}\right)_{n=1}^{\infty} \in \bar{B}_{\ell_{q}}\right\}
$$

so that $T$ is $p$-compact.

Now, if we take a sequence $\left(x_{n}\right)_{n=1}^{\infty} \in \ell_{p}\left(\ell_{p}\right)$ such that $T\left(\bar{B}_{\ell_{q}}\right) \subset p-c o\left\{\left(x_{n}\right)_{n=1}^{\infty}\right\}$, hence in particular $\left\{T\left(e_{n}\right): n \in \mathbb{N}\right\} \subset p-c o\left\{\left(x_{n}\right)_{n=1}^{\infty}\right\}$, then the calculation in the 
proof of Proposition 5.2 shows that

$$
\sum_{n=1}^{\infty}\left|a_{n}\right|^{p} \leq \sum_{n=1}^{\infty}\left\|x_{n}\right\|_{p}^{p}=\left\|\left(x_{n}\right)_{n=1}^{\infty}\right\|_{p}^{p}
$$

and hence $\left\|\left(a_{n}\right)_{n=1}^{\infty}\right\|_{p} \leq\left\|\left(x_{n}\right)_{n=1}^{\infty}\right\|_{p}$. Therefore $k_{p}(T) \geq\left\|\left(a_{n}\right)_{n=1}^{\infty}\right\|_{p}>R$.

The above result also shows the difficulty of getting a factorization of $p$-compact operators of norm $\leq 1$ through $p$-compact operators of norm $\leq 1$ (see the factorization results on $p$-compact operators in [4] and [16]).

\section{ACKNOWLEDGEMENTS}

The authors thank the referee for the careful reading of, and helpful suggestions for, our manuscript.

The research for this paper was started while the second and fourth authors were visiting the Department of Mathematical Sciences at Kent State University. They thank this department for its kind hospitality.

\section{REFERENCES}

[1] Richard M. Aron, Manuel Maestre, and Pilar Rueda, p-compact holomorphic mappings (English, with English and Spanish summaries), Rev. R. Acad. Cienc. Exactas Fís. Nat. Ser. A Math. RACSAM 104 (2010), no. 2, 353-364, DOI 10.5052/RACSAM.2010.22. MR.2757246 (2012e:46093)

[2] Richard M. Aron and Pilar Rueda, p-compact homogeneous polynomials from an ideal point of view, Function spaces in modern analysis, Contemp. Math., vol. 547, Amer. Math. Soc., Providence, RI, 2011, pp. 61-71, DOI 10.1090/conm/547/10807. MR2856480 (2012k:46057)

[3] Richard M. Aron and Pilar Rueda, Ideals of homogeneous polynomials, Publ. Res. Inst. Math. Sci. 48 (2012), no. 4, 957-969, DOI 10.2977/PRIMS/93. MR2999547

[4] Yun Sung Choi and Ju Myung Kim, The dual space of $\left(\mathcal{L}(X, Y), \tau_{p}\right)$ and the p-approximation property, J. Funct. Anal. 259 (2010), no. 9, 2437-2454, DOI 10.1016/j.jfa.2010.06.017. MR2674121 (2011i:46015)

[5] J. M. Delgado, E. Oja, C. Piñeiro, and E. Serrano, The p-approximation property in terms of density of finite rank operators, J. Math. Anal. Appl. 354 (2009), no. 1, 159-164, DOI 10.1016/j.jmaa.2008.12.047. MR2510426 (2010d:46018)

[6] J. M. Delgado and C. Piñeiro, p-compact sets and p-summing evaluation maps, Math. Nachr., to appear.

[7] J. M. Delgado, C. Piñeiro, and E. Serrano, Operators whose adjoints are quasi p-nuclear, Studia Math. 197 (2010), no. 3, 291-304, DOI 10.4064/sm197-3-6. MR2607494(2011g:47041)

[8] J. M. Delgado, C. Piñeiro, and E. Serrano, Density of finite rank operators in the $B a-$ nach space of p-compact operators, J. Math. Anal. Appl. 370 (2010), no. 2, 498-505, DOI 10.1016/j.jmaa.2010.04.058. MR.2651671 (2011j:46025)

[9] Seán Dineen, Complex analysis on infinite-dimensional spaces, Springer Monographs in Mathematics, Springer-Verlag London, Ltd., London, 1999. MR1705327 (2001a:46043)

[10] Alexandre Grothendieck, Produits tensoriels topologiques et espaces nucléaires (French), Mem. Amer. Math. Soc. 1955 (1955), no. 16, 140. MR0075539 (17,763c)

[11] Silvia Lassalle and Pablo Turco, On p-compact mappings and the p-approximation property, J. Math. Anal. Appl. 389 (2012), no. 2, 1204-1221, DOI 10.1016/j.jmaa.2011.12.058. MR2879290(2012m:46022)

[12] Joram Lindenstrauss and Lior Tzafriri, Classical Banach spaces. I, Sequence spaces; Ergebnisse der Mathematik und ihrer Grenzgebiete, Vol. 92, Springer-Verlag, Berlin-New York, 1977. MR0500056 (58 \#17766)

[13] Jorge Mujica, Complex analysis in Banach spaces, Holomorphic functions and domains of holomorphy in finite and infinite dimensions; Notas de Matemática [Mathematical Notes], 107, North-Holland Mathematics Studies, vol. 120, North-Holland Publishing Co., Amsterdam, 1986. MR842435 (88d:46084) 
[14] R. Ryan, Applications of topological tensor products to infinite dimensional holomorphy, Trinity College, Ph.D. Thesis, Dublin, 1980.

[15] Raymond A. Ryan, Introduction to tensor products of Banach spaces, Springer Monographs in Mathematics, Springer-Verlag London, Ltd., London, 2002. MR.1888309 (2003f:46030)

[16] Deba P. Sinha and Anil K. Karn, Compact operators whose adjoints factor through subspaces of $l_{p}$, Studia Math. 150 (2002), no. 1, 17-33, DOI 10.4064/sm150-1-3. MR.1893422 (2003g:46016)

[17] Deba Prasad Sinha and Anil Kumar Karn, Compact operators which factor through subspaces of $l_{p}$, Math. Nachr. 281 (2008), no. 3, 412-423, DOI 10.1002/mana.200510612. MR 2392123 (2009j:46037)

Department of Mathematical Sciences, Kent State University, Kent, Ohio 44242

E-mail address: aron@math.kent.edu

Department of Mathematics, Faculty of Sciences and Arts, Yildiz Technical UniverSity, Davutpaşa, 34210 Esenler, İstanbul, Turkey

E-mail address: ercalis@yahoo.com.tr

Departamento de Análisis Matemático, Universidad de Valencia, Doctor Moliner 50, 46100 Burjasot (VAlencia), Spain

E-mail address: domingo.garcia@uv.es

Departamento de Análisis Matemático, Universidad de Valencia, Doctor Moliner 50, 46100 Burjasot (VAlencia), Spain

E-mail address: manuel.maestre@uv.es 